\title{
3D Silicon Transformation using Hydrogen Annealing
}

\author{
Ming-Chang M. Lee and Ming C. Wu \\ Department of Electrical Engineering, University of California at Los Angeles \\ Los Angeles, CA 90095-1594
}

\begin{abstract}
Fabricating smooth, round structures in silicon is challenging by conventional processing methods. In this paper, we report on a novel 3D shaping technique for producing microspheres, microdisk toroids, submicron wires, and circular beams in Si using hydrogen annealing. Microspheres with $1 \mu \mathrm{m}$ radius, submicron wires with $0.5 \mu \mathrm{m}$ radius, and a microdisk toroid with $0.2 \mu \mathrm{m}$ toroidal radius have been successfully demonstrated.
\end{abstract}

\section{INTRODUCTION}

Thermal annealing in hydrogen ambient has been extensively used in the IC industry as an in situ cleaning step to remove native oxide for silicon epitaxy[1]. In addition, it can reduce surface roughness of as-etched silicon to levels comparable to commercial polished wafers [2][3]. It is believed that heated hydrogen facilitates $\mathrm{Si}$ migration at temperatures much lower than the melting point of $\mathrm{Si}\left(1414^{\circ} \mathrm{C}\right)$. Due to this mass transport effect, a sharp corner on an etched step can be rounded by annealing. Recently, Sato et al. demonstrated several types of buried voids by annealing etched $\mathrm{Si}$ trenches in pure hydrogen at $1100^{\circ} \mathrm{C}$ [4]. However, most of the previous studies focused on the transformation of deep trenches [5]. The deformation of mesa structures on different substrates has not been examined yet.

In this paper, we investigate the effect of hydrogen annealing on the sidewall profiles of microstructures on silicon-on-insulator (SOI). Circular beams and microspheres are fabricated by heating rectangular beams and cylinders, while submicron wires and toroidal microdisks are manufactured by annealing partially released lines and disks on thin SOI.

\section{PRINCIPLE}

The dissociative adsorption of hydrogen on silicon surface increases by many orders of magnitude when the substrate is heated at high temperature [6]. At such temperature, the reaction of hydrogen with silicon enables silicon atoms to migrate on the surface. For features with sharp edges, this mechanism, which is similar to reflow, leads to rounded corners and reduced surface roughness to minimize the surface energy. This is illustrated in Figures 1, which shows the cross-sectional profiles of a comb structure (1- $\mu \mathrm{m}$ fingers with $1-\mu \mathrm{m}$ spacing and $2.5-\mu \mathrm{m}$ height) etched on bulk Si before and after hydrogen annealing. Rounding of sharp corners was clearly observed. The structures remain monocrystalline after annealing.

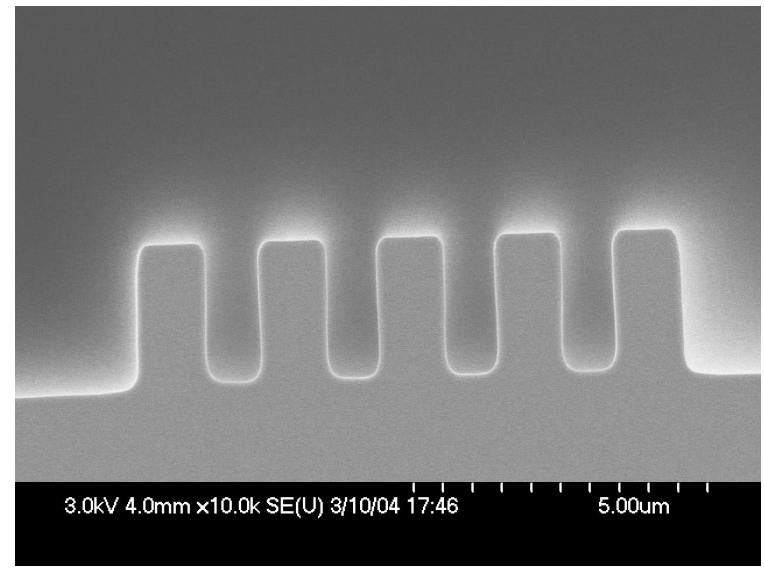

(b)

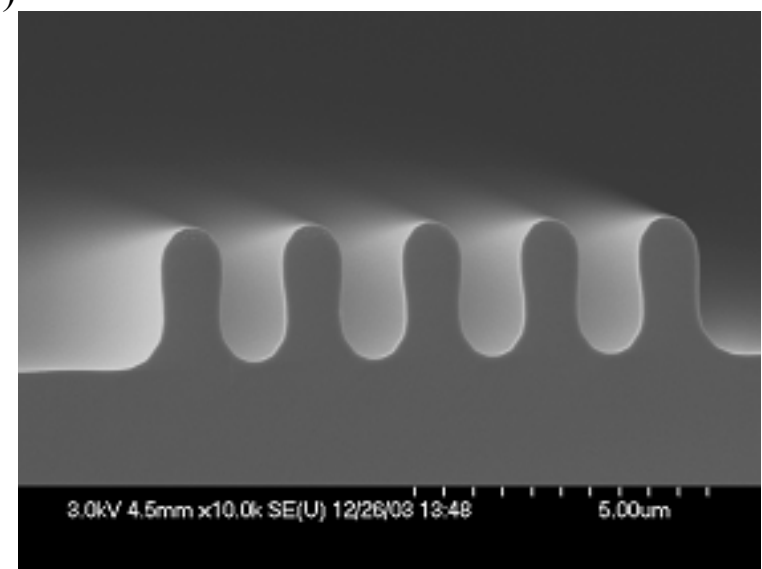

Figure 1 SEM cross-sectional views of comb patterns etched on bulk silicon (a) before and (b) after hydrogen annealing $\left(1100^{\circ} \mathrm{C}\right.$ and 10 Torr).

The surface migration depends on the annealing temperature, the gas pressure, and the reaction time. To investigate the temperature dependence, a $2.5-\mu \mathrm{m}$-tall mesa was etched on a bulk silicon substrate. Then, the wafer was annealed (ASM Episilon II single wafer epitaxial reactor) in pure hydrogen at 10 Torr for 5 minutes. Sharp corners at both the top and the bottom of the mesa became rounded. Figure 2 displays the radius of curvature at the corners versus annealing temperature. It increases from $0.59 \mu \mathrm{m}$ for annealing temperature of $1000^{\circ} \mathrm{C}$ to $0.86 \mu \mathrm{m}$ at $1100^{\circ} \mathrm{C}$. Higher temperatures produce more rounded corners. 


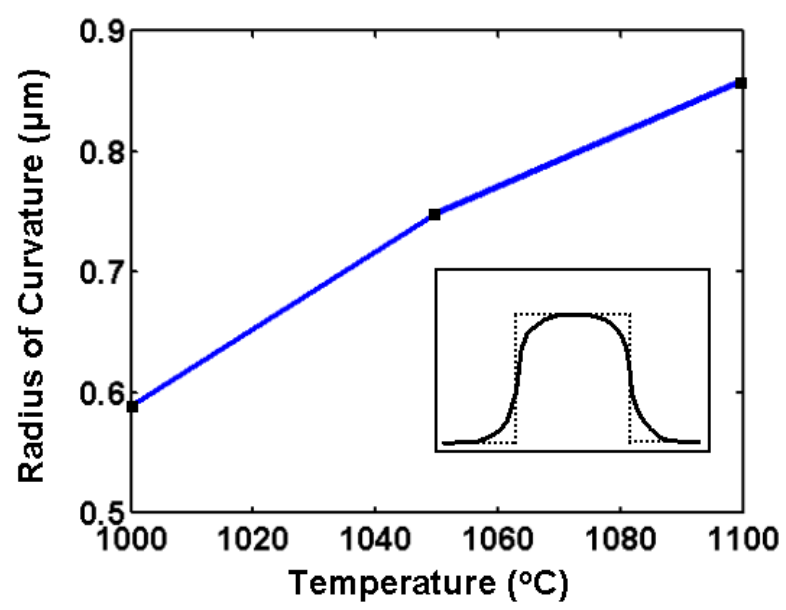

Figure 2. The radius of curvature on the corner of a mesa structure after annealing for 5 minutes at 10 Torr versus annealing temperature.

The sidewall profile produced by hydrogen annealing also depends on bottom interface of the structure. Figure 3 compares the cross sections of the annealed steps on bulk silicon and SOI. The SOI wafer was etched to expose the buried oxide, while the bulk Si wafer was time-etched to the same depth. Then, both wafers were annealed in pure hydrogen at $1100{ }^{\circ} \mathrm{C}, 10$ Torr for 5 minutes. In the bulk silicon sample (Fig. 3(a)), the annealed step exhibits both convex (top) and concave (bottom) corners. In the SOI wafer (Fig. 3(b)), both corners are convex. Furthermore, a slit developed between the etched $\mathrm{Si}$ and the $\mathrm{SiO}_{2}$ due to undercut at the interface. Similar undercut has been observed previously [7]. The undercut can be understood by examining the chemical reactions at the silicon-silicon dioxide interface:

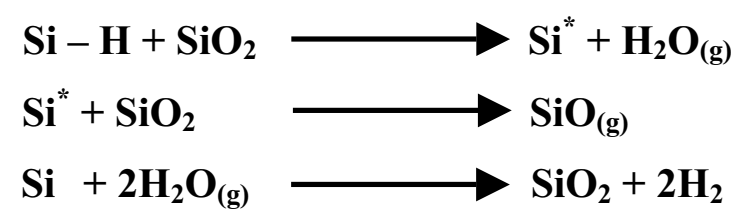

In Eq. (1), the hydrogen atoms attached on silicon react with silicon dioxide to yield water vapor and adsorbed silicon. These silicon atoms are prone to react with silicon dioxide to form volatile $\mathrm{SiO}$. Some of the water vapor re-oxides the exposed silicon to form silicon dioxide. As shown in Eq. (2) and (3), both $\mathrm{Si}$ and $\mathrm{SiO}_{2}$ are consumed at the interface. This phenomenon explains the existence of the slit at the interface. The width of the slit is typically between 0.1 to $0.2 \mu \mathrm{m}$. The length of the undercut region increases with decreasing pressure or increasing temperature.

The undercut at the interface partially release the etched $\mathrm{Si}$ mesa, and after subsequent annealing, a convex corner similar to that at the top of the mesa was observed at the interface, as shown in Fig. 3(b). We can exploit this phenomenon to make circular cross-sectional profiles with very smooth surfaces from rectangular silicon features on SOI substrates.

(a)

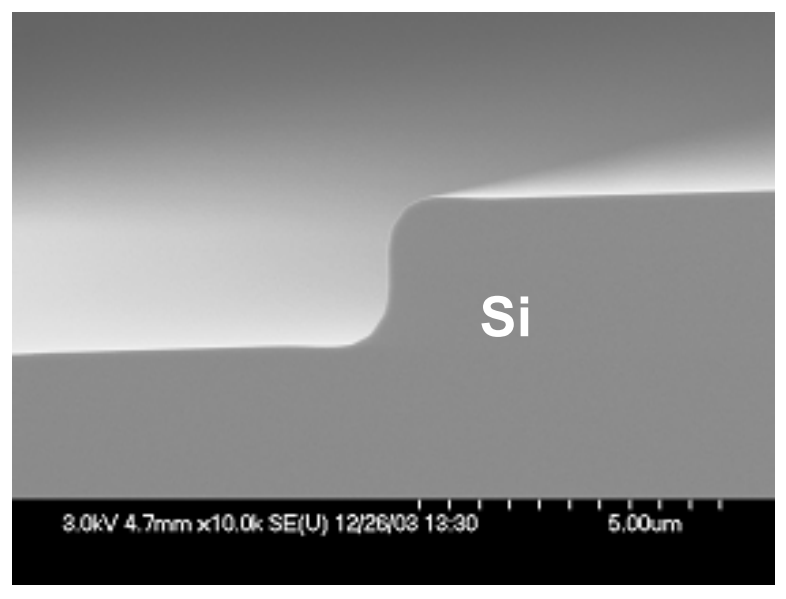

(b)

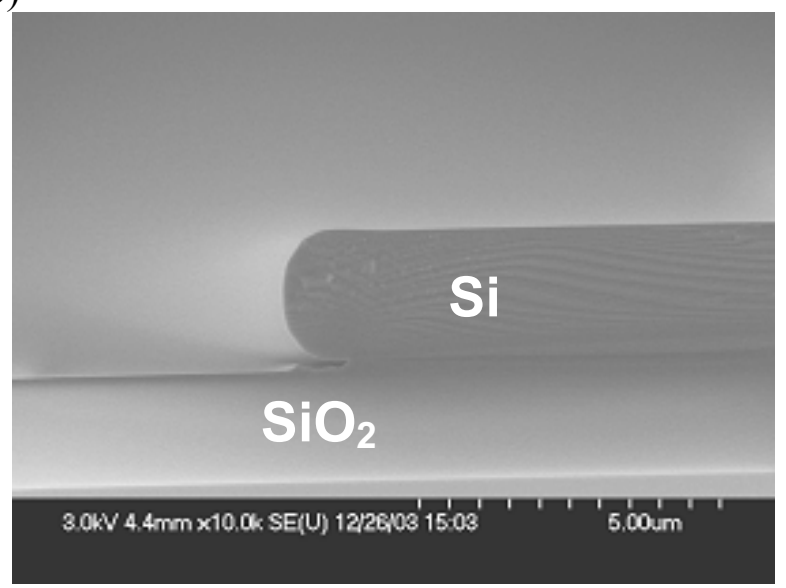

Figure 3 SEM pictures of step profiles on (a) a silicon substrate and (b) an SOI wafer. Both wafers have the same step height and were annealed at $1100^{\circ} \mathrm{C}$ and 10 Torr for five minutes. In SOI wafer, the edge of the $\mathrm{Si}$ mesa is partially released from the $\mathrm{SiO}_{2}$, resulting in a convex bottom corner.

\section{EXPERIMENTAL DETAIL}

\section{A. Circular Beams and Microspheres}

Circular beams were fabricated on SOI wafers with $1.5-\mu \mathrm{m}-$ thick Si films. First, a microbeam array was patterned by lithography. The widths of these lines are comparable to the thickness of the Si film. In this experiment, 1- $\mu \mathrm{m}$ lines with $1-\mu \mathrm{m}$ spacing were employed. After lithography, the structures were dryetched down to the buried oxide with vertical sidewalls. Before thermal annealing, the as-etched sample was dipped in diluted hydrofluoric (HF) acid to remove residual oxide, which could create surface pits during hydrogen annealing [8]. Finally, the sample was annealed in pure hydrogen at $1100^{\circ} \mathrm{C}$ and 10 Torr for five minutes.

Figure 4(a) shows the scanning electron microscopy (SEM) picture of the microbeams after hydrogen annealing. The rectangular beams became circular beams with rounded tips. The beams were released due to the undercut effect described earlier. The microbeam array was anchored to a large-area pad shown on the left of the picture. 
Microspheres were created using a similar process. First, an array of circular cylinders with $1-\mu \mathrm{m}$ radii were created by photolithography and dry etching. The sample is then annealed in pure hydrogen. Figure 4(b) shows the SEM micrograph of the microspheres after hydrogen annealing. The annealing time is controlled to prevent complete release of the microspheres.

(a)

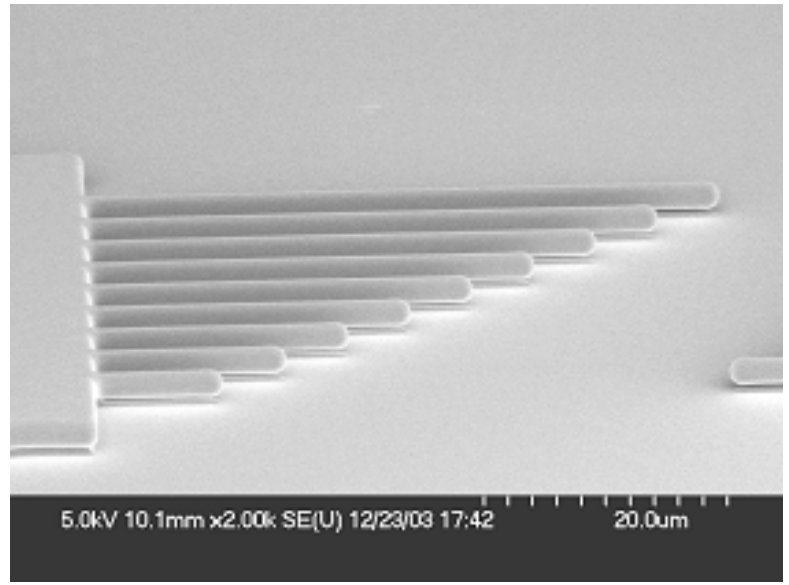

(b)

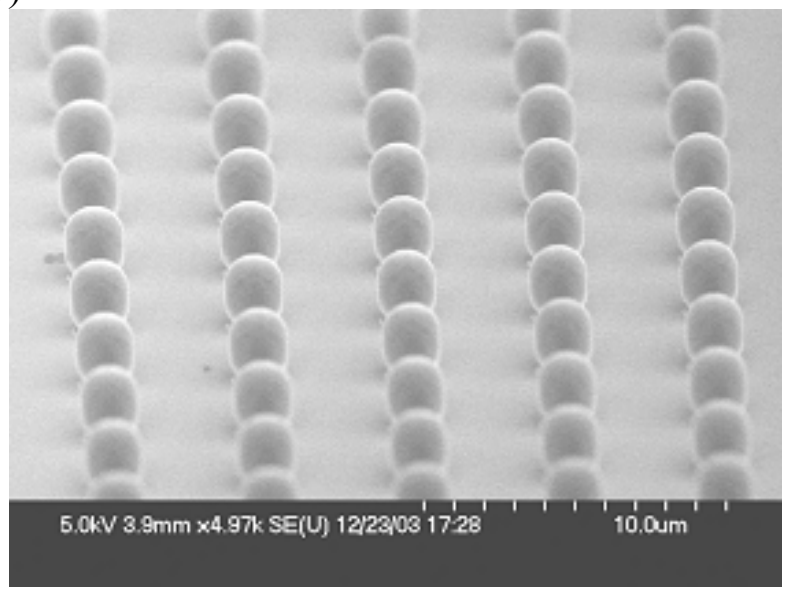

Figure 4(a). Circular microbeams and (b). microspheres. The radius of the microsphere and the diameter of the microbeam is 1 um. Both SEM pictures were taken from an oblique view. The features are made on SOI.

\section{B. Submicron wire and Toroidal Microdisk}

For suspended thin silicon structures, the morphology changes more dramatically, especially at the edges, after hydrogen annealing. Silicon atoms tend to accumulate around the edge to reduce the total surface energy but still maintain a monocrystalline lattice structure.

To demonstrate this effect, an SOI with $0.2-\mu \mathrm{m}$-thick Si film and 3- $\mu \mathrm{m}$-thick buried oxide was patterned to form an array of 1$\mu \mathrm{m}$-wide lines with $1 \mu \mathrm{m}$ spacing. The top and the cross-sectional views of the lines are shown in Fig. 5(a). After releasing in buffered oxide etch (BOE), this array was annealed in hydrogen ambient at $1100^{\circ} \mathrm{C}$ for five minutes. The SEM images are shown in Figure 5(b). The cross-sections of the Si beams were transformed from $0.2 \times 1 \mu \mathrm{m}^{2}$ rectangles to $0.5-\mu \mathrm{m}$-diameter circles. Note that the cross-sectional areas are conserved during the annealing process. Using this technique, sub-micron features can be made without advanced lithography.

(a)

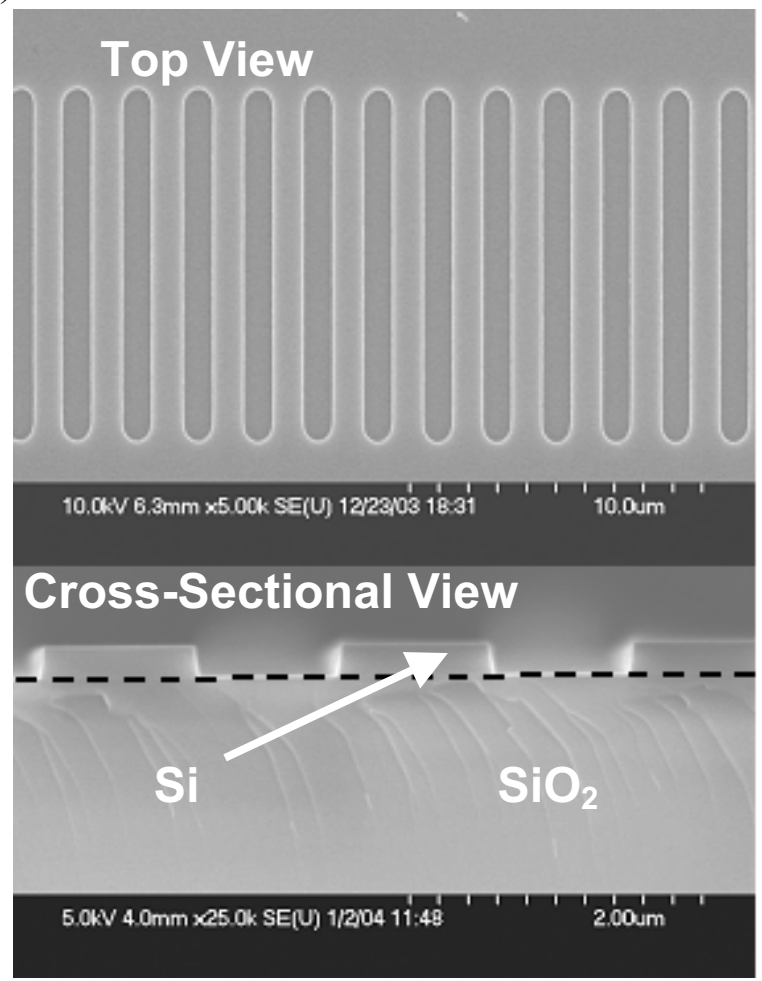

(b)
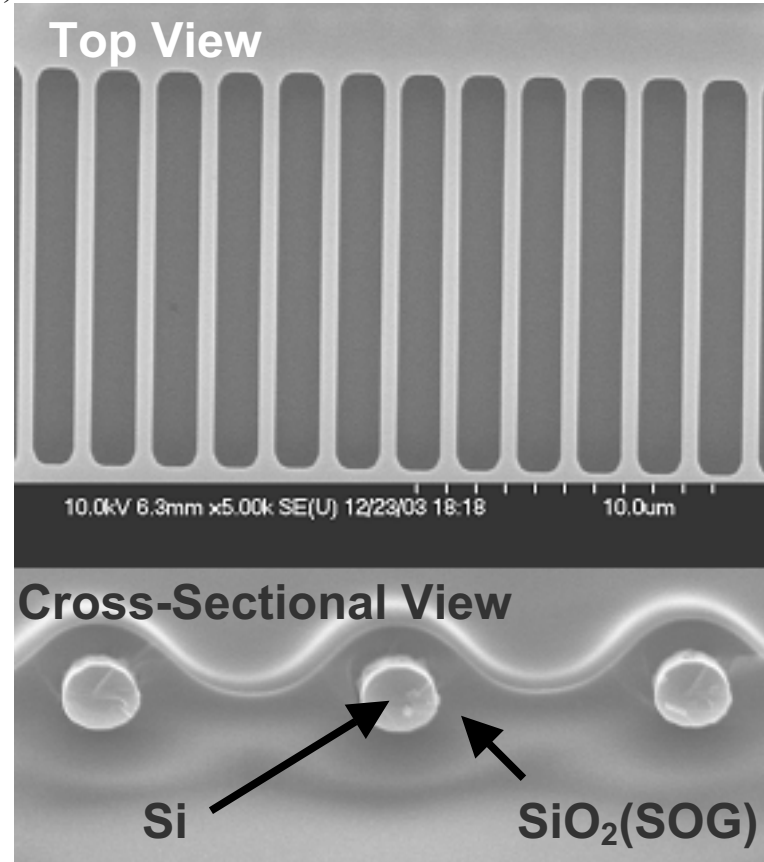

5.0kV $3.9 \mathrm{~mm}$ ×e5.0k SE(U) 12/23/03 $16: 32$ 
Figure 5.. Top- and cross-sectional views of the microbeam array (a) before and (b) after annealing. The SOG (spin-on-glass) in (b) was coated after annealing to hold the suspended structure.

For thin SOI structures with large areas (diameter $>1 \mu \mathrm{m}$ ), the migration of $\mathrm{Si}$ atoms happens primarily at the edges. As a result, a thick rim is formed on the edges of the structure. This can be exploited to make Si microtoroid structures. Circular disks with $10-\mu \mathrm{m}$ radii were first patterned on $0.2-\mu \mathrm{m}$-thick SOI wafers. The disks were partially released in HF to create overhangs at the edges. After annealing in hydrogen, the microdisk was transformed into a toroidal structure with a very smooth surface. Figure 6(a) shows the SEM of a 10- $\mu$ m-radius microtoroid disk. Figure 6(b) shows the cross-sectional view of the toroid. The edges of the disk was not only rounded but also expanded in the vertical direction. The toroidal radius is $200 \mathrm{~nm}$. The microtoroid disks offer several advantages for optical microresonator applications[9].

(a)

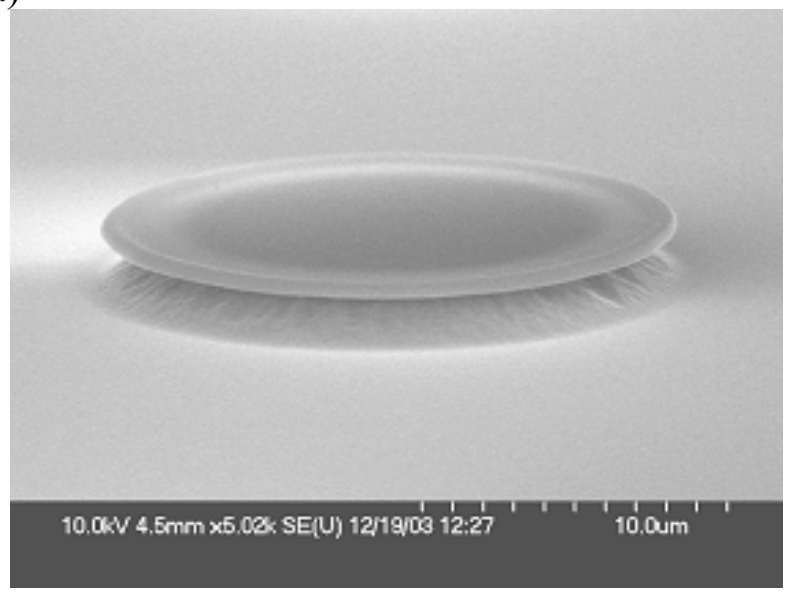

(b)

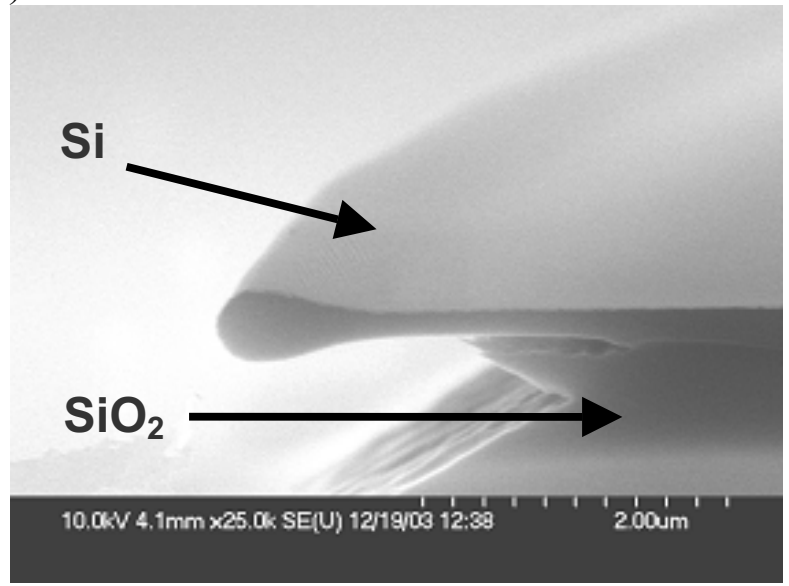

Figure 6. (a) A toroidal structure after hydrogen annealing. (b) A close-up view of the disk cross section. Thickness around the edge is expanded.

\section{CONCLUSIONS}

In summary, we have demonstrated a 3D silicon transformation method using hydrogen annealing. By controlling process temperature, hydrogen pressure, and annealing time, the radius of curvature on the sidewall profile can be engineered. Circular microbeams, microspheres, submicron Si wires, and microtoroid structures have been successfully demonstrated.

\section{ACKNOWLEDGEMENT}

This project is supported by DARPA Optoelectronics Center (\#MDA972-00-1-0019) and the CS-WDM programs (\#MDA97202-1-0019).

\section{REFERENCES}

[1] K. Morishima, et al., Jpn. Pat., No. JP03123027, 1991

[2] Nobuhiko Sato and Takao Yonehara, "Hydrogen Annealed Silicon-on-insulator", Applied Physics Letter, Vol. 65, No. 15, Oct. 1994, pp.1924 1926.

[3] Yang-Kyu Choi, Leland Chang, Pushkar Ranade, Jeong-Soo Lee, Daewon Ha, Sriram Balasubramanian, Aditya Agarwal, Mike Ameen, Tsu-Jae King, and Jeffery Boker, "FinFET Process Refinements of Improved Mobility and Gate Work Function Engineering", International Electron Devices Meeting. Technical Digest, 2002, pp259 262

[4] Tsutomu Sato, Nobutoshi Aoki, Ichiro Mizushima, and Yoshitaka Tsunashima, "A New Substrate Engineering for the Formation of Empty Space in Silicon (ESS) Induced by Silicon Surface Migration", IEDM 1999, pp. 517 520

[5] Hitoshi Kuribayashi, Reiko Hiruta, Ryosuke Shimizu, Koichi Sudoh, and Hiroshi Iwasaki, "Shape Transformation of Silicon Trenches during Hydrogen Annealing", J. Vac. Sci. Technol. A 21(4), Jul/Aug, 2003, pp 1279-1283.

[6] Peter Bratu and Ulrich Hofer, "Photon-Assisted Sticking of Molecular Hydrogen on Si(111)-(7X7)", Physical Review Letters, Vol. 74, No. 9, Feb. 1995, pp 1625-1628

[7] S. T. Liu, L. Chan, and J. O. Borland, "Reaction Kinetics of $\mathrm{SiO} 2 / \mathrm{Si}(100)$ Interface in $\mathrm{H} 2$ Ambient in A Reduced Pressure Epitaxial Reactor", Proc. Electrochem. Soc., 87-88, 1987, pp428 434

[8] Hitoshi Habuka, Hitoshi Tsunoda, Masanori Mayusumi, Nato Tate, and Masatake Katayama, "Roughness of Silicon Surface Heated in Hydrogen Ambient", J. Electrochem. Soc., Vol. 142, No. 9, Sep. 1995, pp. 3092-3098

[9] D. K. Armanl, T. J. Klappenberg, S. M. Splllane and K. J. Vahala, "Ultra-high-Q Toroid Microcavity on A Chip", Nature, Vol. 421, 27 Feb., 2003, pp.926 928 